\title{
Nonsteroidal anti-inflammatory drug gastropathy: new avenues for safety
}

This article was published in the following Dove Press journal:

Clinical Interventions in Aging

28 May 2011

Number of times this article has been viewed

\section{Sanford H Roth}

Arizona Research and Education, Arthritis Laboratory, Arizona State University, Phoenix, AZ, USA
Correspondence: Sanford H Roth Arizona Research and Education, 683I North 58th Place, Paradise Valley, AZ 85253, USA

Tel + I 4809480504

$\mathrm{Fax}+\mathrm{I} 4809480504$

Email sroth16@cox.net

\begin{abstract}
Chronic oral or systemic nonselective nonsteroidal anti-inflammatory drug (NSAID) therapy, ubiquitously used by physicians to treat osteoarthritis-associated pain, is associated with a wide range of symptomatic adverse events, the most frequent and serious of which is gastropathy. Although cardiovascular and renal problems are a very real concern, they are significantly less frequent. These complications can be life-threatening in at-risk populations such as older adults, who are common users of long-term oral systemic NSAID therapy. Topical NSAID formulations deliver effective doses of analgesics directly to the affected joints, thereby limiting systemic exposure and potentially the risk of systemic adverse events, such as gastropathy and serious cardiovascular events. There are currently two topical NSAIDs approved by the US Food and Drug Administration for osteoarthritis-associated pain, as well as for the signs and symptoms of osteoarthritis. This review discusses the relative safety, and the gastrointestinal, cardiovascular, and renal risks of chronic oral or systemic NSAID therapy and topical NSAID formulations in patients with osteoarthritis.
\end{abstract}

Keywords: NSAIDs, osteoarthritis, topical administration, synovial fluid, peptic ulcer, Helicobacter pylori

\section{Introduction}

Osteoarthritis is a chronic, degenerative joint disorder characterized by destruction of articular cartilage, synovial inflammation, and subchondral bone alterations. ${ }^{1}$ It is the most common type of arthritis, affecting approximately 27 million people in the US. ${ }^{1}$ Optimal management of osteoarthritis requires both pharmacologic and nonpharmacologic approaches, with nonsteroidal anti-inflammatory drugs (NSAIDs) frequently selected as the mainstay of pharmacologic treatment. ${ }^{2}$ Although NSAIDs may be the most effective analgesic for many patients with osteoarthritis, when administered orally they confer significant gastrointestinal, cardiovascular, and renal risks. ${ }^{2,3}$ Elderly patients, who constitute the greatest population of patients with osteoarthritis, are at high risk for developing gastrointestinal adverse events, that range from mild dyspepsia or abdominal discomfort to potentially life-threatening complications, such as perforation and hemorrhage. ${ }^{4-6}$ This range of events, known as "NSAID gastropathy", has been recognized for more than two decades as an important complication of longterm NSAID treatment. Despite concomitant administration of medications such as proton pump inhibitors and prostaglandins (misoprostol), this condition persists today in many patients who require NSAID therapy. Optimizing safety in these individuals, who often must take NSAID therapy for extended periods of time, is critical. ${ }^{5,7}$ 
Because of the significant adverse events associated with oral or systemic NSAIDs, it is imperative to seek pharmacologic options with more favorable benefit-risk ratios. Safety concerns are even greater in special populations, such as older adults and those with gastrointestinal, cardiovascular, or renal risk factors. Therefore, the issue of reducing systemic exposure has special relevance in such populations. ${ }^{8}$ This article reviews recent studies evaluating the relative safety of oral NSAID formulations and strategies for balancing the risks of NSAIDs against their well established efficacy in individuals with osteoarthritis.

\section{Mechanisms of action and available formulations}

The analgesic, anti-inflammatory, and antipyretic effects of NSAIDs are a result of the ability of these agents to inhibit cyclo-oxygenase (COX) enzymes, which catalyze the conversion of arachidonic acid to prostaglandins, which are fatty acids involved in the generation of pain, fever, and inflammation. ${ }^{7,9}$ Currently available NSAIDs can be divided into two categories, ie, nonselective NSAIDs, which inhibit both COX-1 and COX-2, and COX-2 selective NSAIDs.

The COX-1 isoenzyme is widely and constitutively expressed in many tissues. ${ }^{9} 10$ It plays an important role in renal and gastrointestinal function, and is located on monocytes and platelets, in the endothelium, and on renal collecting tubules and seminal vesicles. ${ }^{9}$ These expression patterns explain the gastrointestinal and renal adverse events observed with the use of nonselective NSAIDs, in that COX-1 inhibition reduces the cytoprotective effects of this enzyme on gastrointestinal function and renal physiology. ${ }^{9}$ Furthermore, COX-1 inhibition prevents the formation of proaggregatory thromboxane $A_{2}$, which can result in decreased platelet aggregation, and thereby occlusive thrombi, but also can lead to excessive bleeding. ${ }^{9,11}$

In contrast with COX-1, the COX-2 isoenzyme is predominantly involved in pathophysiologic states, such as inflammation and tumorigenesis, and appears substantially more prevalent in injured or inflamed tissues. ${ }^{9,10}$ Found in higher concentrations in the kidney, brain, and reproductive organs, COX-2 is mainly expressed in parenchymal cells. ${ }^{10} \mathrm{COX}-2$ inhibition suppresses some inflammatory mediators involved in the progression of atherogenesis and ischemic myocardial damage, and appears to alter the balance between antithrombotic prostacyclin and prothrombotic thromboxane $\mathrm{A}_{2}$, increasing the risk of thrombosis. ${ }^{9,11}$ COX-2 selective NSAIDs, notably celecoxib, have a recognized dose-related risk of cardiovascular events, but nonselective NSAIDs, such as diclofenac or ibuprofen, also confer cardiovascular risk. ${ }^{12}$

\section{Oral NSAID therapy in osteoarthritis: key safety issues}

The most ubiquitous and problematic adverse event associated with oral (systemic) NSAIDs is gastropathy, which confers a significant risk of decreased work time, disability, and medical costs. Increased risk of mortality from gastrointestinal bleeding, stomach, and bowel perforations is also of significant concern. Thus, although cardiovascular and renal vascular complications pose significant risks, they are comparatively less frequent than gastrointestinal complications. All three categories of events are associated with significant morbidity and mortality, and necessitate careful selection of patients for oral NSAID therapy to minimize risk.

\section{Gastrointestinal adverse events}

The most common adverse events associated with oral (systemic) NSAID therapy are those of the gastrointestinal system. ${ }^{13}$ A recent international, multicenter study of 3293 consecutive candidates for NSAID treatment of osteoarthritis showed that $86.6 \%$ were at increased gastrointestinal risk, and $22.3 \%$ were considered at high risk for gastrointestinal events. ${ }^{14}$ The risk of gastric events increases with age, rising precipitously at age 60 years. ${ }^{15}$ Additional risk factors for gastrointestinal toxicity include history of ulcers (particularly bleeding ulcers), high NSAID dose, Helicobacter pylori infection (which increases ulcer risk by approximately 1.8-fold), and concurrent use of antiplatelet agents and aspirin, both of which are commonly used by older adults. ${ }^{15}$ NSAIDs and $H$. pylori have synergistic effects on risk; in a meta-analysis of 16 studies involving 1625 NSAID users, the odds ratio (OR) for peptic ulcer disease in $H$. pyloripositive NSAID users was 61.1 (95\% confidence interval [CI] 9.98-373), compared with H. pylori-negative nonusers. ${ }^{16}$

Both nonselective and COX-2 selective NSAIDs are associated with some elevated gastrointestinal risk. However, the risk is significantly greater for nonselective NSAIDs. ${ }^{13,17}$ Oral nonselective NSAIDs can cause serious upper and lower gastrointestinal effects, including bleeding, perforation, obstruction, stricture, anemia, and hypoalbuminemia. ${ }^{8}$ Of patients receiving nonselective NSAID therapy, 10\%-30\% develop peptic ulcers; ${ }^{13}$ a study in more than 2000 patients hospitalized for peptic ulcer demonstrated that the mortality rate in patients using nonselective NSAIDs was approximately $35 \%$, and was double the rate of that in patients not taking NSAIDs. ${ }^{17}$ More common gastrointestinal adverse 
events, occurring in 5\%-20\% of NSAID-treated patients, include dyspepsia, diarrhea, and other gastric disturbances. Dyspeptic symptoms do not always correlate with development of ulcers, which in many cases have no discernible symptoms. ${ }^{5,13}$ Although associated with lower morbidity and mortality than perforations, ulcers, and bleeds, dyspeptic symptoms are distressing to many patients, frequently leading to costly symptomatic therapies and diagnostic studies, and may negatively affect adherence. ${ }^{13}$

Current measures to reduce the risk of gastrointestinal events when using oral NSAID therapy include concomitant use of agents such as misoprostol, as well as agents from the proton pump inhibitor class to suppress NSAID-associated increases in gastric acid secretion. ${ }^{13}$ Data from comparative studies have demonstrated that proton pump inhibitors are therapeutically superior to misoprostol, a prostaglandin $\mathrm{E}_{1}$ analog, for this purpose. ${ }^{13,18}$ A strong evidence base exists for use of proton pump inhibitors with NSAIDs to protect against both short-term and long-term gastrointestinal effects. ${ }^{13}$ For example, in one study in which a proton pump inhibitor was added to naproxen plus low-dose (cardioprophylactic) aspirin, endoscopic findings were comparable with those in patients on treatment with celecoxib plus low-dose aspirin. ${ }^{19}$ However, long-term use of proton pump inhibitors is also associated with risks, including a dose-related increase in risk of osteoporotic hip fractures among older adults. ${ }^{20}$ As with other multidrug regimens, the addition of a proton pump inhibitor to NSAID-based therapy increases the cost to the patient, as well as the risk of nonadherence. ${ }^{5,21}$ Nonadherence to proton pump inhibitors among patients receiving oral NSAID therapy is particularly problematic, because the most serious gastric adverse events associated with oral NSAID therapy are often asymptomatic. ${ }^{5,13}$ Patients with no symptoms may assume that the proton pump inhibitor is unnecessary and may be more likely to be noncompliant, when they may be at high risk for gastrointestinal bleeding.

In addition to direct drug costs and adherence, gastropathy adds increased costs to the health care system. ${ }^{22}$ It is estimated that more than 100,000 hospitalizations and 10,000-20,000 deaths each year in the US can be attributed to NSAID-related complications. ${ }^{23}$ The costs of excess hospitalizations have been estimated at $\$ 4$ billion annually, ${ }^{24}$ not to mention the indirect costs of NSAID gastropathy, such as lost productivity. ${ }^{25}$ Oral NSAIDs are likely to have more cost associated with adverse events than topical NSAIDs. ${ }^{26}$ In a randomized trial by Castelnuovo et al comparing the costeffectiveness of topical and oral ibuprofen for knee pain, the total cost of drugs was lower in the topical group. The initial lower cost of oral NSAID therapy was displaced by the higher cost of other drugs to treat gastrointestinal and cardiovascular side effects. ${ }^{27}$ Rahme et al conducted a literature review to summarize data that evaluated the direct costs of NSAIDrelated gastrointestinal adverse effects worldwide. The results showed that the events attributable to nonselective NSAIDs were substantial and that their costs far exceeded the cost of the nonselective NSAID itself. ${ }^{28}$

The level of gastrointestinal risk varies with the specific oral NSAID used. Agents with long half-lives, such as piroxicam, are associated with greater risk of gastrointestinal bleeding and perforation because, in the face of lower renal excretion in the elderly, cumulative systemic NSAID exposure is much greater over time. ${ }^{29}$ Patient factors also modulate risk, in that older adults, especially those with a history of ulcers or concurrent use of aspirin, steroids, or anticoagulants, are at increased risk for these gastrointestinal events. ${ }^{5,8}$ The shrunken gastric microvasculature in older individuals increases their risk for ulcer bleeds, even in the absence of other risk factors. ${ }^{5}$ The risk of gastrointestinal adverse events can be reduced by the use of COX-2 selective agents, although their selectivity and impact on overall risk diminishes at increased doses. ${ }^{30,31}$ In addition, these agents are themselves associated with adverse effects, such as an increased risk of thrombotic cardiovascular events. ${ }^{32,33}$

\section{Cardiovascular adverse events}

Both COX-2 selective and nonselective oral (systemic) NSAIDs confer significant cardiovascular risks, although these events occur far less frequently compared with gastropathy. ${ }^{2,12,34}$ It has been postulated that the cardiovascular effects of NSAIDs result from an imbalance between the effects of thromboxane and prostacyclin on the endothelium. ${ }^{8}$ Based on the relative rates of cardiovascular adverse events with oral NSAIDs, five characteristics have emerged as key mediators of cardiovascular risk potential, ie, COX-2 selectivity, dose, longer plasma half-life, effect on blood pressure, and interaction with concomitant aspirin that may potentially reduce the cardioprotective (antiplatelet) effects of the drug. ${ }^{34}$

Rofecoxib, a COX-2 selective agent, was withdrawn from the global market in 2004 after it was shown to increase the risk of acute myocardial infarction and other cardiovascular events significantly. ${ }^{8}$ Level of risk varies among the oral NSAIDs that remain on the market, including nonselective NSAIDs. Data from a study that evaluated cardiovascular outcomes in Medicare patients who were receiving oral NSAID therapy demonstrated that, among the nonselective 
NSAIDs, ibuprofen was associated with the highest rate of adverse cardiovascular outcomes (11.7 per 100 personyears), followed by diclofenac ( 9.3 per 100 person-years), and naproxen ( 8.5 per 100 person-years). ${ }^{35}$

\section{Renal adverse events}

In addition to gastrointestinal and cardiovascular risks, all oral NSAIDs also confer a serious risk of renal adverse events. ${ }^{3}$ Although comparative data are limited, some studies suggest that fenoprofen and indomethacin may have greater nephrotoxicity than the other nonselective NSAIDs. ${ }^{3}$ Renal adverse events associated with oral NSAIDs include electrolyte retention, reduced glomerular filtration, nephritic syndrome, renal papillary necrosis, hyperkalemia, and chronic renal failure. ${ }^{3}$ Although renal adverse events related to NSAIDs are rare in the population at large, their likelihood increases dramatically in high-risk patients, such as older adults, especially those with concurrent conditions, including underlying volume depletion, renal insufficiency, congestive heart failure, and diabetes. ${ }^{3}$ Significant increases in blood urea nitrogen and creatinine clearance have been observed in older patients, even after discontinuation of NSAIDs. ${ }^{3}$

\section{Osteoarthritis guidelines}

The adverse events associated with use of oral (systemic) NSAIDs are well established and have been studied in both clinical and observational trials. Guidelines for the management of osteoarthritis provide suggestions for minimizing the risks of oral NSAID use. The American Geriatrics Society guidelines recommend that nonselective NSAIDs and COX-2 selective NSAIDs be used rarely and with extreme caution, only in patients who have been carefully selected to minimize risk of gastrointestinal, cardiovascular, or renal adverse events, and only when safer alternative therapies have failed. ${ }^{36}$ These guidelines caution that older individuals taking nonselective NSAIDs and individuals on aspirin therapy taking COX-2 selective agents should take a proton pump inhibitor or misoprostol for gastroprotection. The American College of Gastroenterology cautions against the use of oral NSAID therapy in patients at high risk for gastrointestinal bleeding, even in conjunction with misoprostol and a proton pump inhibitor, and strongly recommends that safer alternatives to oral NSAID therapy be utilized. ${ }^{37}$ The American Academy of Orthopedic Surgeons guidelines suggest that patients with symptomatic osteoarthritis of the knee and increased gastrointestinal risk (age $\geq 60$ years, comorbid medical conditions, history of peptic ulcer disease, history of gastrointestinal bleeding, concurrent use of corticosteroids, and/or anticoagulants) receive one of the following analgesics for pain: acetaminophen (not to exceed $4 \mathrm{~g} /$ day), a topical NSAID, a nonselective oral NSAID plus a gastroprotective agent, or a COX-2 inhibitor. ${ }^{38}$

\section{Topical NSAIDs in osteoarthritis: potential for risk reduction}

Nonselective NSAIDs are available as oral formulations which produce systemic levels of drug and as topical formulations which deliver drug to the local target tissue and result in significantly lower levels of systemic absorption relative to oral therapy. ${ }^{7}$ Whereas plasma drug concentrations after administration of topical NSAIDs are comparatively low (generally $<10 \%$ of that after oral administration), local drug concentrations in target tissues are high. ${ }^{7,39}$ Lower plasma concentrations of active drug after application of topical NSAIDs appear to be associated with a lower risk of systemic adverse events compared with oral formulations. ${ }^{39}$ However, data vary among the available topical agents. Only diclofenac topical solution has been compared with oral diclofenac in head-to-head trials, in which diclofenac topical solution established comparable efficacy and a lower incidence of systemic adverse events compared with oral diclofenac. ${ }^{40,41}$

Topical diclofenac, in gel and solution formulations, is the only topical NSAID currently approved by the Food and Drug Administration for the management of osteoarthritis. ${ }^{42,43}$ Although other topical salicylates are available over the counter to treat joint pain and were recommended in the American College of Rheumatology guidelines in 2000, these agents do not have the evidence base of diclofenac to support their use, possibly because of poor skin penetration properties, ${ }^{2,42,43}$ and being nonacetylated salicylates with absent prostaglandin inhibition, they are weak analgesics. ${ }^{44}$

In clinical practice, topical NSAIDs are used for the many patients with osteoarthritis who require NSAID therapy but desire or need to minimize the risk of systemic adverse events or who prefer topical to oral dosing. ${ }^{42,45} \mathrm{~A}$ recent review by Haroutiunian et al reported that the incidence of severe gastrointestinal adverse events is three times lower with topical diclofenac compared with oral diclofenac, and that its systemic effects across the board were generally infrequent and did not differ from placebo in the great majority of trials. ${ }^{7}$ Another recent systematic review by Makris et al evaluated the safety of topical NSAIDs in older adults with osteoarthritis. These authors concluded that the use of topical NSAIDs resulted in fewer severe gastrointestinal adverse events compared with oral NSAIDs in older adults. ${ }^{46}$ Both of these reviews included data from a wide range of 
topical NSAID preparations, including agents not currently available in the US. ${ }^{7,46}$

In the US, diclofenac is available topically as a gel and as a solution for the management of osteoarthritis. ${ }^{42,43}$ A number of placebo-controlled studies have examined the safety and efficacy of these two topical diclofenac formulations individually.

\section{Topical diclofenac solution with dimethylsulfoxide}

Several randomized controlled trials have shown that topical diclofenac solution with a dimethylsulfoxide penetration enhancer (Pennsaid ${ }^{\circledR}$, Mallinckrodt Inc, Hazelwood, MO) is effective and well tolerated in the treatment of signs and symptoms of knee osteoarthritis. Three vehicle-controlled studies, ranging in length from 4-12 weeks, established the ability of topical diclofenac solution to improve pain scores, physical function, patient global assessment, stiffness, and pain on walking. ${ }^{47-49}$ The most common adverse events were local skin irritation, such as dryness. ${ }^{47-49}$ Skin dryness may be due to the potential of dimethylsulfoxide to dissolve skin lipids, and may be countered by the application of skin emollients. However, the effect of emollients on skin dryness has not been specifically evaluated. ${ }^{40}$

Topical diclofenac solution is also unique among the diclofenac formulations available in the US, in that it has been compared with oral diclofenac in head-to-head trials including osteoarthritis of the knee. In two 12-week activecontrolled studies comparing topical diclofenac solution with oral diclofenac, topical diclofenac produced pain relief in patients with osteoarthritis comparable with pain relief experienced with oral diclofenac. ${ }^{40,41}$ In addition, topical diclofenac solution was associated with fewer systemic adverse events compared with oral diclofenac. In a study by Tugwell et al, patients treated with topical diclofenac solution had a significantly lower incidence of gastrointestinal adverse events compared with patients receiving oral diclofenac, including dyspepsia ( $15 \%$ versus $26 \%, P=0.001$ ), abdominal pain $(12 \%$ versus $22 \%, P=0.0008)$, nausea $(8 \%$ versus $13 \%, P=0.04)$, and diarrhea ( $9 \%$ versus $17 \%, P=0.001) .{ }^{41}$ Incidence of abnormal liver function (aspartate transaminase, $2 \%$ versus $10 \%, P=0.0001$; alanine transaminase, $5 \%$ versus $17 \%, P<0.0001)$ and altered laboratory parameters (eg, creatinine clearance, an indicator of renal effects) also occurred less frequently with topical diclofenac solution. ${ }^{41}$

Topical diclofenac solution demonstrated similar safety advantages in a study by Simon et al, in which only $6.5 \%$ of patients treated with topical diclofenac solution experienced gastrointestinal adverse events, compared with $23.8 \%$ of those receiving oral diclofenac (dyspepsia, 2.6\% versus $4.0 \%$; abdominal pain, $3.2 \%$ versus $7.3 \%$; nausea, $0 \%$ versus $2.0 \%$; diarrhea, $1.3 \%$ versus $4.6 \%){ }^{40}$ The incidence of cardiovascular events was comparably low among patients receiving oral diclofenac and treated with topical diclofenac solution $(<2 \%)$, as was the incidence of hypertension $(1.3 \%) .{ }^{40}$ Very large, longer-term studies would likely be needed to detect a difference in the incidence of cardiovascular effects between topical diclofenac solution and oral diclofenac therapy.

\section{Topical diclofenac gel}

Diclofenac gel 1\% (Voltaren ${ }^{\circledR}$ gel, Endo Pharmaceuticals Inc, Chadds Ford, PA) has been studied in placebo-controlled studies to determine efficacy and safety in the treatment of pain associated with osteoarthritis. Diclofenac gel was significantly more effective than placebo in three studies (one 3-week and two 12-week), as would be expected..$^{50-52}$ In all three trials, the incidence of gastrointestinal adverse events was similar between patients treated with diclofenac gel (ranging from $0 \%$ to $5.9 \%$ ) and patients treated with placebo (ranging from $1.7 \%$ to $5.0 \%) .^{50-52}$ One study noted a higher incidence of cardiovascular events among patients treated with diclofenac gel compared with placebo (four events versus one event), although these events were deemed unrelated to treatment. ${ }^{51}$ The rates of gastrointestinal adverse events occurring in these studies of diclofenac gel are substantially lower than those with oral diclofenac treatment in other trials including osteoarthritis. ${ }^{40,41}$ Observed discontinuation rates due to adverse reactions were only slightly higher for diclofenac gel versus placebo ( $5 \%$ versus $3 \%$ ). ${ }^{53}$ The most common cause for discontinuation was application site reactions. ${ }^{53}$ The incidence of adverse events demonstrated in a long-term trial (up to 1 year) was similar to incidence in the placebo-controlled studies. ${ }^{53}$ In this long-term trial, application site dermatitis was observed in $11 \%$ of patients and led to discontinuation for $6 \%$ of patients. ${ }^{53}$

\section{Summary}

Osteoarthritis remains a prevalent yet too often unsafely treated disease, due to the well documented, systemic adverse events associated with the ubiquitous use of oral (systemic) NSAID therapy. ${ }^{8,13}$ It is estimated that almost half of the adult population may develop symptomatic osteoarthritis of the knee by the age of 85 years. ${ }^{54}$ Oral NSAIDs, widely used in the treatment of osteoarthritis, are associated with significant gastrointestinal adverse events, which the introduction of COX-2 selective NSAIDs has not completely eliminated., ${ }^{3,13}$ NSAID gastropathy remains a silent threat, despite various efforts to manage it. The frequent gastric complications have 
introduced a tremendous cost burden due to the widespread chronic use of systemic NSAIDs in the elderly. ${ }^{55}$ In addition to gastrointestinal adverse events, oral nonselective NSAID therapy and treatment with COX-2 selective agents also increase the risk of cardiovascular events in a dose-dependent manner. ${ }^{12}$

Therefore, clinicians need strategies to reduce the risk of NSAID-related systemic adverse events as part of an overall approach to the management of osteoarthritis. One important possible risk-reduction strategy is the use of topical NSAIDs, which have been shown to be effective in osteoarthritis, but with a generally reduced risk of systemic adverse events compared with oral NSAIDs. ${ }^{48}$ All of the topical NSAIDs available in the US contain diclofenac and vary in formulation, use of penetration enhancers, and evidence base to support their use. Only topical diclofenac solution has been studied in direct comparison with oral diclofenac. In these studies, topical diclofenac solution exhibited comparable efficacy and a reduced incidence of systemic adverse events versus oral diclofenac. ${ }^{40,41}$ Several reviews and meta-analyses have shown topical NSAIDs to have significant safety advantages to minimize gastrointestinal risk while improving tolerability in high-risk patients. Rates of gastrointestinal adverse events and ulcers, perforation, or bleeding have been much higher with oral nonselective NSAIDs and celecoxib than with topical diclofenac formulations. ${ }^{52,56,57}$ In addition, there have been two studies in patients with osteoarthritis of the knee showing similar efficacy between topical diclofenac solution and oral diclofenac. Additional head-to-head studies in patients with osteoarthritis are needed to determine whether diclofenac gel performs similarly relative to oral diclofenac.

Because of the differences in evidence base and in potential, as yet unknown, differences in actual efficacy and systemic adverse events among the topical diclofenac formulations, generalizations about the relative risk of topical versus oral NSAIDs should be minimized in favor of specific discussion of the relevant risks of each topical diclofenac formulation. Due to their demonstrated efficacy, safety, and potential to reduce systemic adverse events when compared with oral NSAIDs, topical NSAIDs can be considered important additions to the armamentarium for the treatment of osteoarthritis. ${ }^{43}$ Future long-term studies on chronic use will likely reveal that topical NSAIDs are a safer alternative to oral systemic NSAIDs, and may relieve high-risk elderly patients from common gastrointestinal and cardiorenal NSAID-related adverse events, as well as diminish systemic NSAID levels in the elderly.

\section{Disclosure}

Dr Roth is a current stakeholder within Transdel Pharmaceuticals, and serves as a consultant and speaker for Covidien.

\section{References}

1. Centers for Disease Control and Prevention. Arthritis-related statistics, August 2010. Available from: http://www.cdc.gov/arthritis/data_ statistics/arthritis_related_stats.htm. Accessed October 19, 2010.

2. American College of Rheumatology Subcommittee on Osteoarthritis Guidelines. Recommendations for the medical management of osteoarthritis of the hip and knee: 2000 update. Arthritis Rheum. 2000; 43(9): 1905-1915.

3. Harirforoosh S, Jamali F. Renal adverse effects of nonsteroidal antiinflammatory drugs. Expert Opin Drug Saf. 2009;8(6):669-681.

4. Roth SH. NSAID gastropathy. A new understanding. Arch Intern Med. 1996;156(15):1623-1628

5. Roth SH. Nonsteroidal antiinflammatory drug gastropathy: We started it, why don't we stop it? J Rheumatol. 2005;32(7):1189-1191.

6. Sun CHE, Roth SH, Mitchell CS, Englund DW. Upper gastrointestinal disease in rheumatoid arthritis. Dig Dis. 1974;19(5):405-410.

7. Haroutiunian S, Drennan DA, Lipman AG. Topical NSAID therapy for musculoskeletal pain. Pain Med. 2010;11(4):535-549.

8. Chan FK. Primer: Managing NSAID-induced ulcer complications balancing gastrointestinal and cardiovascular risks. Nat Clin Pract Gastroenterol Hepatol. 2006;3(10):563-573.

9. Rao P, Knaus EE. Evolution of nonsteroidal anti-inflammatory drugs (NSAIDs): cyclooxygenase (COX) inhibition and beyond. $J$ Pharm Pharm Sci. 2008;11(2):81S-110s.

10. Rouzer CA, Marnett LJ. Cyclooxygenases: Structural and functional insights. J Lipid Res. 2009;50 Suppl:S29-S34.

11. Weir MR, Sperling RS, Reicin A, Gertz BJ. Selective COX-2 inhibition and cardiovascular effects: A review of the rofecoxib development program. Am Heart J. 2003;146(4):591-604.

12. McGettigan P, Henry D. Cardiovascular risk and inhibition of cyclooxygenase: A systematic review of the observational studies of selective and nonselective inhibitors of cyclooxygenase 2. JAMA. 2006;296(13): $1633-1644$.

13. Lazzaroni M, Porro GB. Management of NSAID-induced gastrointestinal toxicity: Focus on proton pump inhibitors. Drugs. 2009;69(1): 51-69.

14. Lanas A, Tornero J, Zamorano JL. Assessment of gastrointestinal and cardiovascular risk in patients with osteoarthritis who require NSAIDs: The LOGICA study. Ann Rheum Dis. 2010;69(8):1453-1458.

15. Bardou M, Barkun AN. Preventing the gastrointestinal adverse effects of nonsteroidal anti-inflammatory drugs: From risk factor identification to risk factor intervention. Joint Bone Spine. 2010;77(1):6-12.

16. No authors listed. H. pylori eradication in NSAID-associated ulcers. Drug Ther Bull. 2005;43(5):37-40.

17. Thomsen RW, Riis A, Munk EM, Norgaard M, Christensen S, Sorensen HT. 30-day mortality after peptic ulcer perforation among users of newer selective COX-2 inhibitors and traditional NSAIDs: A population-based study. Am J Gastroenterol. 2006;101(12):2704-2710.

18. Davies NM, Longstreth J, Jamali F. Misoprostol therapeutics revisited. Pharmacotherapy. 2001;21(1):60-73.

19. Goldstein JL, Cryer B, Amer F, Hunt B. Celecoxib plus aspirin versus naproxen and lansoprazole plus aspirin: A randomized, doubleblind, endoscopic trial. Clin Gastroenterol Hepatol. 2007;5(10): 1167-1174.

20. Yang YX, Lewis JD, Epstein S, Metz DC. Long-term proton pump inhibitor therapy and risk of hip fracture. JAMA. 2006;296(24):2947-2953.

21. Osterberg L, Blaschke T. Adherence to medication. $N$ Engl J Med. 2005;353(5):487-497.

22. Johnson RE, Hornbrook MC, Hooker MC, Woodson GT, Shneidman R. Analysis of the costs of NSAID-gastropathy. Experience in a US health maintenance organisation. Pharmacoeconomics. 1997;12(1):76-88. 
23. Wolfe MM, Lichtenstein DR, Singh G. Gastrointestinal toxicity of nonsteroidal antiinflammatory drugs. $N$ Engl J Med. 1999;340(24): 1888-1899.

24. Smalley WE, Griffin MR, Fought RL, Ray WA. Excess costs from gastrointestinal disease associated with nonsteroidal anti-inflammatory drugs. J Gen Intern Med. 1996;11(8):461-469.

25. Fendrick AM, Scheiman JM. Decision analytic modeling and NSAID gastropathy: Could we be more sensitive? J Gen Intern Med. 2000;15(6):430-431.

26. Cross PL, Ashby D, Harding G, et al. Are topical or oral ibuprofen equally effective for the treatment of chronic knee pain presenting in primary care: A randomized controlled trial with patient preference study. BMC Musculoskelet Disord. 2005;6:55.

27. Castelnuovo E, Cross P, Mt-Isa S, Spencer A, Underwood M; on behalf of the TOIB study team. Cost-effectiveness of advising the use of topical or oral ibuprofen for knee pain; the TOIB study. Rheumatology (Oxford). 2008;47(7):1077-1081.

28. Rahme E, Barkun AN, Adam V, Bardou M. Treatment costs to prevent or treat upper gastrointestinal adverse events associated with NSAIDs. Drug Saf. 2004;27(13):1019-1042.

29. Massó González EL, Pastrignani P, Tacconelli S, García Rodríguez LA. Variability among nonsteroidal antiinflammatory drugs in risk of upper gastrointestinal bleeding. Arthritis Rheum. 2010;62(6):1592-1601.

30. Deeks JJ, Smith LA, Bradley MD. Efficacy, tolerability, and upper gastrointestinal safety of celecoxib for treatment of osteoarthritis and rheumatoid arthritis: Systematic review of randomised controlled trials. BMJ. 2002;325(7365):619.

31. Silverstein FE, Faich G, Goldstein JL, et al. Gastrointestinal toxicity with celecoxib vs nonsteroidal anti-inflammatory drugs for osteoarthritis and rheumatoid arthritis: The CLASS study: A randomized controlled trial. Celecoxib Long-term Arthritis Safety Study. JAMA. 2000;284(10):1247-1255.

32. Wright JM. The double-edged sword of COX-2 selective NSAIDs. CMAJ. 2002;167(10):1131-1137.

33. Mukherjee D, Nissen SE, Topol EJ. Risk of cardiovascular events associated with selective COX-2 inhibitors. JAMA. 2001;286(8):954-959.

34. Farkouh ME, Greenberg BP. An evidence-based review of the cardiovascular risks of nonsteroidal anti-inflammatory drugs. Am J Cardiol. 2009;103(9):1227-1237.

35. Solomon DH, Glynn RJ, Rothman KJ, et al. Subgroup analyses to determine cardiovascular risk associated with nonsteroidal antiinflammatory drugs and coxibs in specific patient groups. Arthritis Rheum. 2008;59(8):1097-1104.

36. American Geriatrics Society Panel on the Pharmacological Management of Persistent Pain in Older Persons. Pharmacological management of persistent pain in older persons. J Am Geriatr Soc. 2009;57(8): 1331-1346.

37. Lanza FL, Chan FK, Quigley EM; Practice Parameters Committee of the American College of Gastroenterology. Guidelines for prevention of NSAID-related ulcer complications. Am J Gastroenterol. 2009;104(3): 728-738.

38. American Academy of Orthopaedic Surgeons. Treatment of Osteoarthritis of the Knee (Non-Arthoplasty) Full Guideline. Rosemont, IL: American Academy of Orthopedic Surgeons; 2008.

39. Moore RA, Derry S, McQuay HJ. Topical agents in the treatment of rheumatic pain. Rheum Dis Clin North Am. 2008;34(2):415-432.
40. Simon LS, Grierson LM, Naseer Z, Bookman AA, Zev Shainhouse J. Efficacy and safety of topical diclofenac containing dimethyl sulfoxide (DMSO) compared with those of topical placebo, DMSO vehicle and oral diclofenac for knee osteoarthritis. Pain. 2009;143(13):238-245.

41. Tugwell PS, Wells GA, Shainhouse JZ. Equivalence study of a topical diclofenac solution (Pennsaid) compared with oral diclofenac in symptomatic treatment of osteoarthritis of the knee: A randomized controlled trial. J Rheumatol. 2004;31(10):2002-2012.

42. Altman R, Barkin RL. Topical therapy for osteoarthritis: Clinical and pharmacologic perspectives. Postgrad Med. 2009;121(2):139-147.

43. Altman RD. Practical considerations for the pharmacologic management of osteoarthritis. Am J Manag Care. 2009;15(8 Supp1):S236-S243.

44. Roth SH. Salicylates revisited. Are they still the hallmark of antiinflammatory therapy? Drugs. 1988;36(1):1-6.

45. Carnes D, Anwer Y, Underwood M, Harding G, Parsons S; TOIB Study Team. Influences on older people's decision making regarding choice of topical or oral NSAIDs for knee pain: Qualitative study. BMJ. 2008; 336(7636):142-145.

46. Makris UE, Kohler MJ, Fraenkel L. Adverse effects of topical nonsteroidal anti-inflammatory drugs in older adults with osteoarthritis: A systematic literature review. J Rheumatol. 2010;37(6):1236-1243.

47. Bookman AA, Williams KS, Shainhouse JZ. Effect of a topical diclofenac solution for relieving symptoms of primary osteoarthritis of the knee: A randomized controlled trial. CMAJ. 2004;171(4):333-338.

48. Roth SH, Shainhouse JZ. Efficacy and safety of a topical diclofenac solution (PENNSAID) in the treatment of primary osteoarthritis of the knee: A randomized, double-blind, vehicle-controlled clinical trial. Arch Intern Med. 2004;164(18):2017-2023.

49. Baer PA, Thomas LM, Shainhouse Z. Treatment of osteoarthritis of the knee with a topical diclofenac solution: A randomised controlled, 6-week trial [ISRCTN53366886]. BMC Musculoskelet Disord. 2005; $6: 44$.

50. Niethard FU, Gold MS, Solomon GS, et al. Efficacy of topical diclofenac diethylamine gel in osteoarthritis of the knee. J Rheumatol. 2005; 32(12):2384-2392.

51. Barthel HR, Haselwood D, Longley S 3rd, Gold MS, Altman RD. Randomized controlled trial of diclofenac sodium gel in knee osteoarthritis. Semin Arthritis Rheum. 2009;39(3):203-212.

52. Baraf HS, Gold MS, Clark MB, et al. Safety and efficacy of topical diclofenac sodium 1\% gel in knee osteoarthritis: A randomized controlled trial. Phys Sportsmed. 2010;38(2):19-28.

53. Voltaren Gel (diclofenac sodium topical gel) 1\% [prescribing information]. Chadds Ford, PA: Endo Pharmaceuticals Inc.

54. Murphy L, Schwartz TA, Helmick CG, et al. Lifetime risk of symptomatic knee osteoarthritis. Arthritis Rheum. 2008;59(9):1207-1213.

55. Viviane AV, Alan BN. Estimates of costs of hospital say for variceal and nonvariceal upper gastrointestinal bleeding in the United States. Value Health. 2008;11(1):1-3.

56. Niculescu L, Li C, Huang J, Mallen S. Pooled analysis of GI tolerability of 21 randomized controlled trials of celecoxib and nonselective NSAIDs. Curr Med Res Opin. 2009;25(3):729-740.

57. Moore RA, Derry S, Makinson GT, McQuay HJ. Tolerability and adverse events in clinical trials of celecoxib in osteoarthritis and rheumatoid arthritis: Systematic review and meta-analysis of information from company clinical trial reports. Arthritis Res Ther. 2005;7(3): R644-R645.
Clinical Interventions in Aging

\section{Publish your work in this journal}

Clinical Interventions in Aging is an international, peer-reviewed journal focusing on evidence-based reports on the value or lack thereof of treatments intended to prevent or delay the onset of maladaptive correlates of aging in human beings. This journal is indexed on PubMed Central, MedLine, the American Chemical Society's 'Chemical

\section{Dovepress}

Abstracts Service' (CAS), Scopus and the Elsevier Bibliographic databases. The manuscript management system is completely online and includes a very quick and fair peer-review system, which is all easy to use. Visit http://www.dovepress.com/testimonials.php to read real quotes from published authors. 